\title{
APPROXIMATELY INNER DERIVATIONS
}

\author{
OLA BRATTELI, AKITAKA KISHIMOTO and DEREK W. ROBINSON
}

(Dedicated to the memory of Gert K. Pedersen)

\begin{abstract}
Let $\alpha$ be an approximately inner flow on a $C^{*}$-algebra $A$ with generator $\delta$ and let $\delta_{n}$ denote the bounded generators of the approximating flows $\alpha^{(n)}$. We analyze the structure of the set

$$
\mathscr{D}=\left\{x \in D(\delta): \lim _{n \rightarrow \infty} \delta_{n}(x)=\delta(x)\right\}
$$

of pointwise convergence of the generators. In particular we examine the relationship of $\mathscr{D}$ and various cores related to spectral subspaces.
\end{abstract}

\section{Introduction}

The theory of flows on operator algebras has been largely motivated by models of quantum statistical mechanics in which the flow $\alpha$ is usually constructed as the limit of a sequence $\left(\alpha^{(n)}\right)$ of local flows. The latter are typically given by inner one-parameter automorphism groups of the algebra $A$ and the flow is correspondingly called approximately inner. In particular this is the situation for models of quantum spin systems in which the algebra of observables $A$ is a UHF-algebra (see, for example, [9], Chapter 6). An early result of Sakai established [25] that if $\delta$ is the generator of a flow $\alpha$ on a UHF-algebra then there exists an increasing sequence $\left(A_{n}\right)$ of finite dimensional $C^{*}$-subalgebras $A_{n}$ of $A$ such that $A_{n} \subset D(\delta)$ and $\bigcup_{n} A_{n}$ is dense in $A$. Furthermore, there exists a sequence $\left(h_{n}\right)$ of elements $h_{n}$ of the self-adjoint part $A_{s a}$ of $A$ such that $\left.\delta\right|_{A_{n}}=\left.\operatorname{ad}\left(i h_{n}\right)\right|_{A_{n}}$ (see [8], Example 3.2.25). If, in addition, $\bigcup_{n} A_{n}$ is a core for $\delta_{\alpha}$, i.e. $\bigcup_{n} A_{n}$ is dense in $D(\delta)$ in the graph norm, then it follows that $\alpha$ is approximately inner. In particular one has

$$
\lim _{n \rightarrow \infty} \max _{|t| \leq 1}\left\|\alpha_{t}(x)-\alpha_{t}^{(n)}(x)\right\|=0
$$

for all $x \in A$ where $\alpha_{t}^{(n)}=e^{t \delta_{n}}$ with $\delta_{n}=\operatorname{ad}\left(i h_{n}\right)$. This is a consequence of two general results. First the strong convergence (1) of the semigroups is equivalent to strong convergence of the resolvents $\left(\iota \pm \delta_{n}\right)^{-1}$ to $(\iota \pm \delta)^{-1}$ by the 
Kato-Trotter theorem (see, for example, [14], Theorem IX.2.16). Explicitly (1) is equivalent to the condition

$$
\lim _{n \rightarrow \infty}\left\|\left(\iota \pm \delta_{n}\right)^{-1}(x)-(\iota \pm \delta)^{-1}(x)\right\|=0
$$

for all $x$ in a norm-dense subspace of $A$. Secondly, since $\bigcup_{n} A_{n} \subset \mathscr{D}$, where

$$
\mathscr{D}=\left\{x \in D(\delta): \lim _{n \rightarrow \infty} \delta_{n}(x)=\delta(x)\right\},
$$

it follows that $\mathscr{D}$ is a core of $\delta$. In particular the subspaces $(\iota \pm \delta)(\mathscr{D})$ are norm-dense in $A$. Then if $y \in \mathscr{D}$ and $x_{ \pm}=(\iota \pm \delta)(y)$ one has

$$
\lim _{n \rightarrow \infty}\left\|\left(\iota \pm \delta_{n}\right)^{-1}\left(x_{ \pm}\right)-(\iota \pm \delta)^{-1}\left(x_{ \pm}\right)\right\| \leq \lim _{n \rightarrow \infty}\left\|\left(\delta_{n}-\delta\right)(y)\right\|=0 .
$$

Therefore the strong resolvent convergence (2) and the equivalent semigroup convergence (1) is established. Thus the crucial feature in this argument is the core property of $\mathscr{D}$. This follows automatically if $\bigcup_{n} A_{n}$ is a core.

Secondly, note that it does not follow in general from Sakai's construction that $\bigcup_{n} A_{n}$ can be taken to be a core for $\delta$. This was an open problem for many years. The affirmative answer would imply that $\alpha$ is approximately inner, and this was known as the Powers-Sakai conjecture. The problem was finally resolved in the negative in 2000 (see [20], Theorem 1.1). The counterexample is, however, an AF-algebra which is not UHF and the problem still seems to be open for UHF-algebras.

The purpose of this note is to analyze the convergence (1) for flows $\alpha_{t}^{(n)}=$ $e^{i t \delta_{n}}$ and $\alpha_{t}=e^{i t \delta}$ on a general $C^{*}$-algebra $A$ by examining the structure of the set $\mathscr{D}$ defined by (3). In particular we consider the relation between $\mathscr{D}$ and various natural cores of $\delta$.

\section{Cores and spectral subspaces}

Let $\alpha$ be a flow on a $C^{*}$-algebra $A$ with generator $\delta_{\alpha}$. Define the spectral subspace $A^{\alpha}(K)$, for each closed subset $K$ of $\mathrm{R}$, as the Banach subspace spanned by the $x \in A$ for which the conditions $f \in L_{1}(\mathrm{R})$ and supp $\hat{f} \cap K=\emptyset$ imply $\alpha_{f}(x)=0$. (Here $\hat{f}$ denotes the Fourier transform of $f$.) The $\alpha$-spectrum of $x \in A$, denoted by $\operatorname{Sp}_{\alpha}(x)$, is defined to be the smallest closed subset $K$ of $\mathrm{R}$ such that $x \in A^{\alpha}(K)$. Elements with compact $\alpha$-spectra are referred to as geometric elements of $\alpha$ and the subspace of geometric elements is denoted by $A_{G}^{\alpha}$. Explicitly, $A_{G}^{\alpha}=\bigcup_{n=1}^{\infty} A^{\alpha}([-n, n])$. Note that each geometric element is automatically an entire analytic element of $\alpha$. Moreover, $A_{G}^{\alpha}$ is a ${ }^{*}$-subalgebra of $A$ and an $\alpha$-invariant core of $\delta_{\alpha}$. 
The spectrum $\operatorname{Sp}(\alpha)$ of $\alpha$ is defined to be the smallest closed subset $K$ of $\mathrm{R}$ such that $A=A^{\alpha}(K)$. The Connes spectrum $\mathrm{R}(\alpha)$ of $\alpha$ is defined to be the intersection of $\operatorname{Sp}\left(\left.\alpha\right|_{B}\right)$ with $B$ all non-zero $\alpha$-invariant hereditary $C^{*}$-algebras of $A$. It is known that $\mathbf{R}(\alpha)$ is a closed subgroup of $\mathbf{R}$.

Our first result establishes under quite general conditions that the geometric elements of an approximately inner flow are not contained in the subspace $\mathscr{D}$ of convergence.

THEOREM 2.1. Let $A$ be a separable $C^{*}$-algebra and $\alpha$ an approximately inner flow with generator $\delta_{\alpha}$. Suppose there exists a faithful family of $\alpha$-covariant irreducible representations of $A$ and that $\mathrm{R}(\alpha) \neq\{0\}$. Let $\left(h_{n}\right)$ be a sequence of self-adjoint elements of A such that

$$
\lim _{n \rightarrow \infty} \max _{|t| \leq 1}\left\|\alpha_{t}(x)-\operatorname{Ad} e^{i t h_{n}}(x)\right\|=0
$$

for all $x \in A$ and let

$$
\mathscr{D}=\left\{x \in D\left(\delta_{\alpha}\right): \lim _{n \rightarrow \infty} \operatorname{ad} i h_{n}(x)=\delta_{\alpha}(x)\right\} .
$$

Then

$$
\mathscr{D} \not \supset A_{G}^{\alpha} \text {. }
$$

Proof. Under the assumptions of the theorem it follows that there is a faithful family of irreducible representations $\left(\pi, \mathscr{H}_{\pi}\right)$ of $A$ such that the representation

$$
\bar{\pi}=\int_{\mathrm{R}}^{\oplus} d t \pi \circ \alpha_{t}
$$

of $A$ on $L_{2}\left(\mathrm{R} ; \mathscr{H}_{\pi}\right)$ is of type I with centre $L_{\infty}(\mathrm{R})$ if $\mathrm{R}(\alpha)=\mathrm{R}$ and $L_{\infty}\left(\mathrm{R} /\left((2 \pi p)^{-1} \mathrm{Z}\right)\right.$ )) (as a subalgebra of $\left.L_{\infty}(\mathrm{R})\right)$ if $\mathrm{R}(\alpha)=p \mathrm{Z}$. (This is Theorem 1.2 of [18] when $A$ is prime. See Remark 2.3 when $A$ is not prime.) Define a unitary flow $U$ on $L_{2}\left(\mathrm{R} ; \mathscr{H}_{\pi}\right)$ by $U_{t} \xi(s)=\xi(s+t)$. Then $U_{t} \bar{\pi}(x) U_{t}^{*}=$ $\bar{\pi}\left(\alpha_{t}(x)\right)$. We denote by $\bar{\alpha}_{t}$ the weakly continuous flow $t \mapsto \operatorname{Ad} U_{t}$ on $\bar{\pi}(A)^{\prime \prime}$.

Since $A$ is separable, there is a countable faithful family of such irreducible representations. Let $\left(\pi_{i}\right)$ be such a family. Let $q \in \mathrm{R}(\alpha)$ and define $z_{i} \in$ $\bar{\pi}_{i}(A)^{\prime \prime} \cap \bar{\pi}_{i}(A)^{\prime} \subset L_{\infty}(\mathrm{R})$ by $z_{i}(t)=e^{2 \pi i q t}$. Note that $z_{i}$ satisfies $\bar{\alpha}_{t}\left(z_{i}\right)=$ $e^{2 \pi i q t} z_{i}$. Then there is a net $\left(y_{\mu}\right)$ in the unit ball of $A$ such that $\bar{\pi}_{i}\left(y_{\mu}\right)$ converges to $z_{i}$ in the $*$-strong topology for any $i$ and $\pi\left(y_{\mu}\right)$ converges to 0 weakly for any representation $\pi$ disjoint from all $\bar{\pi}_{i}$. We may further suppose that the $\alpha$ spectrum of $y_{\mu}$ decreases to $\{q\}$. Since $A$ is separable and the direct sum of $\bar{\pi}_{i}$ is a representation on a separable Hilbert space, one can choose a sequence $\left(y_{k}\right)$ from the convex combinations of $\left(y_{\mu}\right)$ such that $\left(y_{k}\right)$ is a central sequence, $\bar{\pi}_{i}\left(y_{k}\right)$ converges to $z_{i}$ in the $*$-strong topology, and the $\alpha$-spectrum of $y_{k}$ 
shrinks to $\{q\}$. Since $\bar{\pi}_{i}\left(y_{k} y_{k}^{*}\right)$ converges to the identity for any $i$ and the direct sum of $\bar{\pi}_{i}$ is faithful, one may conclude that $\left\|x y_{k}\right\|$ converges to $\|x\|$ for any $x \in A$ as $k \rightarrow \infty$. We use this fact below.

Assume that $\mathscr{D} \supset A^{\alpha}([-\varepsilon, \varepsilon])$ for an $\varepsilon>0$. Then it follows from the uniform boundedness theorem that there is constant $c>0$ such that

$$
\left\|\left.\operatorname{ad} i h_{m}\right|_{A^{\alpha}([-\varepsilon, \varepsilon])}\right\|<c
$$

for all $m$. Choose $x \in A^{\alpha}([q-\varepsilon / 2, q+\varepsilon / 2])$ such that $\|x\|=1$ where $q \in \mathrm{R}(\alpha)$ satisfies $q>c+\varepsilon$. By the arguments in the previous paragraph one can find a central sequence $\left(y_{k}\right)$ in $A$ such that $\left\|y_{k}\right\| \leq 1, \operatorname{Sp}_{\alpha}\left(y_{k}\right) \subset\langle-q-$ $\varepsilon / 2,-q+\varepsilon / 2\rangle$ and $\bar{\pi}\left(y_{k} y_{k}^{*}\right)$ converges weakly to the identity as $k \rightarrow \infty$. Then $\left\|x y_{k}\right\| \leq 1,\left\|x y_{k}\right\| \rightarrow 1$ and $\operatorname{Sp}_{\alpha}\left(x y_{k}\right) \subset\langle-\varepsilon, \varepsilon\rangle$. Hence $\left\|\operatorname{ad} i h_{m}\left(x y_{k}\right)\right\|<c$ and

$$
\lim _{k \rightarrow \infty}\left\|\operatorname{ad} i h_{m}\left(x y_{k}\right)-\operatorname{ad} i h_{m}(x) y_{k}\right\|=0 .
$$

Since $\lim _{m \rightarrow \infty}\left\|\operatorname{ad} i h_{m}(x)-\delta_{\alpha}(x)\right\|=0$ and $\lim _{k \rightarrow \infty}\left\|\delta_{\alpha}(x) y_{k}\right\|=\left\|\delta_{\alpha}(x)\right\|$ it follows that $\|$ ad $i h_{m}\left(x y_{k}\right)\|>\| \delta_{\alpha}(x) \|-\varepsilon / 2 \geq q-\varepsilon>c$ for all sufficiently large $m$ and $k$. This contradicts the bound $\|$ ad $i h_{m}\left(x y_{k}\right) \|<c$. Therefore $\mathscr{D} \not \supset \bigcup_{n=1}^{\infty} A^{\alpha}([-n, n])$.

REMARK 2.2. The foregoing proof establishes a slightly stronger statement: If $\mathscr{D}$ contains $A^{\alpha}([-\varepsilon, \varepsilon])$ for some $\varepsilon>0$, then $\mathscr{D} \cap A^{\alpha}([q-\varepsilon / 2, q+\varepsilon / 2])=$ $\{0\}$ for all large $q \in \mathrm{R}(\alpha)$.

REMARK 2.3. In Theorem 2.1 we assumed the condition

(i) there exists a faithful family of $\alpha$-covariant irreducible representations of $A$.

The essential requirement is, however, a consequence of the assumption

(ii) there exists a faithful family $\left\{\pi_{i}\right\}$ of irreducible representations of $A$ such that $\bar{\pi}_{i}$ is of type I and the spectrum of $\bar{\alpha}$ on the center $\bar{\pi}_{i}(A)^{\prime \prime} \cap \bar{\pi}_{i}(A)^{\prime}$, which we denote by $\Delta(\pi)$, is $\mathrm{R}(\alpha)$, where $\bar{\pi}_{i}$ and $\bar{\alpha}$ are defined in the above proof.

As we asserted above (i) implies (ii). To confirm this assertion let us define $\mathbf{R}_{2}(\alpha)$ to be the set of $p \in \mathbf{R}$ satisfying: for any non-zero $x \in A$ and any $\varepsilon>0$ there exists an $a \in A^{\alpha}([p-\varepsilon, p+\varepsilon])$ such that $\|a\|=1$ and $\left\|x\left(a+a^{*}\right) x^{*}\right\| \geq$ $(2-\varepsilon)\|x\|^{2}$. It is obvious that $\mathbf{R}_{2}(\alpha)$ is a closed subset of $\mathbf{R}(\alpha)$. Furthermore one can show that the inclusion $R_{2}(\alpha) \supset \bigcap_{\pi \in \mathscr{F}} \Delta(\pi)$ holds for any faithful family $\mathscr{F}$ of irreducible representations of $A$ and the equality holds for some (see [17], Proposition 1). Consider the conditions

(i') $\mathrm{R}_{2}(\hat{\alpha})=\mathrm{R}$ 
and

(ii') $\mathbf{R}_{2}(\alpha)=\mathbf{R}$.

One can show that (i) $\Leftrightarrow\left(\mathrm{i}^{\prime}\right) \Rightarrow$ (ii') $\Leftrightarrow$ (ii). If $A$ is prime all these conditions are equivalent [18]. The equivalences of (i) with (i') and (ii) with (ii') are a kind of duality and these equivalences are straightforward. The only implication which is not explicitly given in the non-prime case seems to be (i') $\Rightarrow$ (ii'). The arguments we adopt here are given in the proof of Theorem 3.3 of [16]. Let $B=A \times{ }_{\alpha} \mathrm{R}$, let $H$ be a discrete subgroup of $\mathrm{R}(\alpha)$, and let $\beta=\left.\hat{\alpha}\right|_{H}$. Then it follows from (i') that there is a faithful family of covariant irreducible representations for $(B \times \beta, \hat{H}, \hat{\beta})$ and it follows from $H \subset \mathrm{R}(\alpha)$ that $H(\hat{\beta})=$ $H$. Let $x \in B \times \times_{\beta} H$. For $p \in H^{\perp}$ and any compact neighborhood $U$ of $p$ in $\mathrm{R}$ one can show from (i') that

$$
\sup \left\{\left\|x\left(a+a^{*}\right) x^{*}\right\|: a \in B^{\hat{\alpha}}(U),\|a\|=1\right\}=2\|x\|^{2} .
$$

Moreover, for $s \in H$ one can show by using Glimm's type of theorem for the compact dynamical system $\left(B \times_{\beta} H, \hat{H}, \hat{\beta}\right)[6]$ that

$$
\sup \left\{\left\|x\left(a+a^{*}\right) x^{*}\right\|: a \in B \lambda(s)\right\}=2\|x\|^{2},
$$

where $H \ni s \rightarrow \lambda(s)$ is the canonical unitary group in the multiplier algebra of $B \times{ }_{\beta} H$ implementing $\beta$. Using these two conditions one can construct a faithful family $\left\{\pi_{i}\right\}$ of irreducible representations of $B \times{ }_{\beta} H$ such that $\pi_{i}$ restricts to an irreducible representation $\rho_{i}$ of $B$ and $\bar{\rho}_{i}$ which is the direct integral of $\rho_{i} \hat{\alpha}_{p}, p \in \mathrm{R}$ is of type I with $\Delta\left(\rho_{i}\right)=H^{\perp}$. Then, by Lemma 5 of [17], the duality implies that $\mathrm{R}_{2}(\alpha) \supset H$. Since $H$ is an arbitrary discrete subgroup of $\mathbf{R}(\alpha)$ one can conclude that $\mathbf{R}_{2}(\alpha)=\mathbf{R}(\alpha)$.

It follows automatically from the assumptions of Theorem 2.1 that one has $\mathscr{D} \not \supset D$ for any core $D$ of $\delta_{\alpha}$ which contains the geometric elements $A_{G}^{\alpha}$. In particular $\mathscr{D}$ cannot contain the analytic elements, or the $C^{\infty}$ elements, of $\alpha$. In addition one cannot construct a core in $\mathscr{D}$ by regularization of the subspace of geometric elements since the following lemma establishes that the subspace is unchanged by regularization with respect to the flow.

Lemma 2.4. Let $A_{G}^{\alpha}$ denote the geometric elements of the flow $\alpha$ on the $C^{*}$-algebra A. Then

$$
\begin{aligned}
A_{G}^{\alpha} & =\left\{\alpha_{f}(x): x \in A_{G}^{\alpha}, f \in L_{1}(\mathrm{R})\right\} \\
& =\operatorname{span}\left\{\alpha_{f}(x): x \in A_{G}^{\alpha}, f \in C_{c}^{\infty}(\mathrm{R})\right\} .
\end{aligned}
$$

Proof. First $x \in A^{\alpha}(K)$ if and only if $g \in L_{1}(\mathrm{R})$ and ( $\left.\operatorname{supp} \hat{g}\right) \cap K=\emptyset$ imply $\alpha_{g}(x)=0$. But if $x \in A^{\alpha}(K)$ and $f \in L_{1}(\mathrm{R})$ then $\alpha_{g}\left(\alpha_{f}(x)\right)=\alpha_{f * g}(x)$. 
Moreover, $\operatorname{supp}(\widehat{f * g}) \subseteq \operatorname{supp} \hat{g}$. Therefore $\alpha_{g}\left(\alpha_{f}(x)\right)=0$ and one deduces that $\alpha_{f}(x) \in A^{\alpha}(K)$. Hence

$$
\left\{\alpha_{f}(x): x \in A_{G}^{\alpha}, f \in C_{c}^{\infty}(\mathrm{R})\right\} \subseteq\left\{\alpha_{f}(x): x \in A_{G}^{\alpha}, f \in L_{1}(\mathrm{R})\right\} \subseteq A_{G}^{\alpha} .
$$

Next if $x \in A^{\alpha}(K)$ and $f, g \in L_{1}(\mathrm{R})$ with $\hat{f}=\hat{g}=1$ on an open neighbourhood $U$ of $K$ then $\alpha_{f}(x)=\alpha_{g}(x)$ (see, for example, [8], Lemma 3.2.38). Replacing $g$ by an approximate identity, with the Fourier transform equal to one on $U$, and taking the limit one deduces that $\alpha_{f}(x)=x$. Therefore

$$
A_{G}^{\alpha}=\left\{\alpha_{f}(x): x \in A_{G}^{\alpha}, f \in L_{1}(\mathrm{R})\right\} .
$$

But the same argument also gives

$$
A_{G}^{\alpha}=\left\{\alpha_{f}(x): x \in A_{G}^{\alpha}, f \in \mathscr{S}(\mathrm{R})\right\}
$$

where $\mathscr{S}(\mathrm{R})$ is the usual Schwartz space. Finally, if $f \in \mathscr{S}(\mathrm{R})$ then it follows from the Rubel-Squires-Taylor factorization theorem [24] (see also [13]) that there exists a finite set of $g_{i} \in C_{c}^{\infty}(\mathrm{R})$ and $h_{i} \in \mathscr{S}(\mathrm{R})$ such that $f=\sum g_{i} * h_{i}$. In particular $\alpha_{f}(x)=\sum \alpha_{g_{i}}\left(y_{i}\right)$ with $y_{i}=\alpha_{h_{i}}(x)$. Therefore

$$
\begin{aligned}
A_{G}^{\alpha} & =\left\{\alpha_{f}(x): x \in A_{G}^{\alpha}, f \in \mathscr{S}(\mathrm{R})\right\} \\
& \subseteq \operatorname{span}\left\{\alpha_{g}(y): y \in A_{G}^{\alpha}, g \in C_{c}^{\infty}(\mathrm{R})\right\} \\
& \subseteq \operatorname{span}\left\{\alpha_{g}(y): y \in A_{G}^{\alpha}, g \in \mathscr{S}(\mathrm{R})\right\}=A_{G}^{\alpha}
\end{aligned}
$$

and the proof is complete.

Despite these observations Example 3.6 illustrates that many approximately inner flows of interest in mathematical physics are such that the subspace of convergence $\mathscr{D}$ contains a dense invariant set of analytic elements.

It is remarkable that under the conditions of Theorem 2.1 convergence of a sequence of bounded derivations on the subspace $A_{G}^{\alpha}$ automatically implies boundedness of the limit derivation, at least if $A$ is prime:

Corollary 2.5. Let $A$ be a separable prime $C^{*}$-algebra and $\alpha$ a flow. Suppose there exists a faithful family of $\alpha$-covariant irreducible representations of $A$ and that $\mathrm{R}(\alpha) \neq\{0\}$.

Suppose that there is a sequence $\left(b_{n}\right)$ of self-adjoint elements of $A$ such that the limits

$$
\delta(x)=\lim _{n \rightarrow \infty} \text { ad } i b_{n}(x)
$$

exist for all $x \in A_{G}^{\alpha}$. Then $x \in A_{G}^{\alpha} \mapsto \delta(x) \in A$ extends to a bounded *-derivation on $A$. 
Proof. It follows from the uniform boundedness theorem that the norm of ad $\left.b_{n}\right|_{A^{\alpha}([-k, k])}$ is bounded as $n \rightarrow \infty$ for each $k$. Hence $\left.\delta\right|_{A^{\alpha}([-k, k]}$ is bounded. Then, by [15], $\delta$ is closable and its closure $\bar{\delta}$ generates a flow $\beta$.

By [19] there is a faithful covariant representation $(\pi, U)$ of $A$ such that the flow $\bar{\alpha}: t \mapsto \operatorname{Ad} U_{t}$ on the factor $M=\pi(A)^{\prime \prime}$ has Connes spectrum $\mathrm{R}(\alpha)$, e.g. $\pi$ may be a type $\mathrm{II}_{\infty}$ representation extending the tracial representation of a UHF algebra (with a non-trivial UHF flow) "embedded" in $A$; here we need $A$ to be prime. Let $f \in L_{1}(\mathrm{R})$ be an integrable real-valued function such that the Fourier transform $\hat{f}$ has compact support and define

$$
\delta_{f}=\int_{\mathrm{R}} d t f(t) \alpha_{t} \delta \alpha_{-t} .
$$

The closure $\bar{\delta}_{f}$ of $\delta_{f}$ is also a generator and we denote by $\beta_{f}$ the flow generated by $\bar{\delta}_{f}$. By [5] the $\alpha$-covariant representation $\pi$ is also $\beta_{f}$-covariant. If $f \in$ $L_{1}(\mathrm{R})$ satisfies $\hat{f}(0)=0$ then $\delta_{f}$ is bounded. Moreover, there is a constant $c>0$ such that $\left\|\delta_{f}\right\| \leq c\|f\|_{1}$ for such $f$.

We fix a positive function $f \in L_{1}(\mathrm{R})$ such that $\operatorname{supp}(\hat{f})$ is compact and $\hat{f}(0)=\int d t f(t)=1$ and define $\delta_{n}$ as the closure of $1 / n \int d t f(t / n) \alpha_{t} \delta \alpha_{-t}$. Define $\Delta_{n}$ to be the weak extension of $x \mapsto \pi \delta_{n}(x)$ on $\pi\left(\mathscr{D}\left(\delta_{n}\right)\right)$, i.e. the generator of the weak extension of the flow on $\pi(A)$ induced by $\delta_{n}$. Since $\left\|\Delta_{n}-\Delta_{1}\right\| \leq 2 c$, we take a limit point $d$ of the sequence of derivations $\Delta_{n}-\Delta_{1}: \pi(A)^{\prime \prime} \rightarrow \pi(A)^{\prime \prime}$ with pointwise weak topology. Thus the limit $d$ is a derivation on $M$. Then by general theory $d$ is an inner derivation on $M$. Hence $\Delta_{1}+d$ is a generator. Since $\Delta_{1}+d$ is a limit point of $\Delta_{n}: \mathscr{D}\left(\Delta_{1}\right) \rightarrow M$ and $\left\|\bar{\alpha}_{t} \Delta_{n}(x)-\Delta_{n} \bar{\alpha}_{t}(x)\right\| \rightarrow 0, x \in M_{G}^{\bar{\alpha}}$ as $n \rightarrow \infty$, we conclude that $\Delta_{1}+d$ commutes with $\bar{\alpha}$. By the arguments originating in [21] we argue that $\Delta_{1}+d$ generates a flow which is a bounded perturbation of a scaled $\bar{\alpha}$ as follows: Let $\gamma$ be the flow on $M$ generated by $\Delta_{1}+d$. We denote by $\bar{\alpha} \otimes \gamma$ the action of $\mathrm{R}^{2}$ on $M$ defined by $(s, t) \mapsto \bar{\alpha}_{s} \gamma_{t}$. Since the spectrum of $\bar{\alpha} \otimes \gamma$ is bounded on each $\{p\} \times \mathbf{R}$ and the Connes spectrum $\mathbf{R}(\bar{\alpha} \otimes \gamma)$ is included in $\mathbf{R}(\alpha) \times \mathbf{R}$, there is a constant $\lambda \in \mathbf{R}$ such that $\mathbf{R}(\bar{\alpha} \otimes \gamma)=\{(p, \lambda p): p \in \mathbf{R}(\alpha)\}$. Since $\operatorname{Sp}(\bar{\alpha} \otimes \gamma)+\mathrm{R}(\bar{\alpha} \otimes \gamma)=\operatorname{Sp}(\bar{\alpha} \otimes \gamma)$, we conclude that $\gamma$ is a bounded perturbation of the flow $t \mapsto \bar{\alpha}_{\lambda t}$ or the flow on $A$ generated by $\delta_{1}$ is a bounded perturbation of the flow $t \mapsto \alpha_{\lambda t}$.

On the other hand define $\delta_{n}^{\prime}$ to be the closure of $n \int d t f(n t) \alpha_{t} \delta \alpha_{-t}$ and $\Delta_{n}^{\prime}$ to be the weak extension of $x \mapsto \pi\left(\delta_{n}^{\prime}(x)\right)$. Then by the same token we have that $\left\|\Delta_{1}-\Delta_{n}^{\prime}\right\| \leq 2 c$. Note that $\mathscr{D}\left(\Delta_{1}\right)=\mathscr{D}\left(\Delta_{n}^{\prime}\right)$ and $\left\|\pi\left(\delta_{1}(x)-\delta_{n}^{\prime}(x)\right)\right\| \leq$ $2 c\|\pi(x)\|$ for $x \in \mathscr{D}\left(\delta_{1}\right)$. We then conclude that there is a derivation $d^{\prime}$ of $M$ such that $\pi\left(\delta_{1}(x)\right)-d^{\prime}(\pi(x))=\pi(\delta(x))$ for $x \in \pi\left(A_{G}^{\alpha}\right)$. Since $A_{G}^{\alpha}$ is dense in $A$, this implies that $d^{\prime}$ leaves $\pi(A)$ invariant. Hence, since $\pi$ is faithful, 
$x \in A_{G}^{\alpha} \mapsto \delta_{1}(x)-\delta(x)$ extends to a derivation of $A$. Combining this with the result in the previous paragraph, the flow $\beta$ (generated by $\bar{\delta}$ ) is a bounded perturbation of $t \mapsto \alpha_{\lambda t}$. If $\lambda \neq 0$, this would imply that $\left.\delta_{\alpha}\right|_{A_{G}^{\alpha}}$ is approximated by inner derivations, which contradicts Theorem 2.1. Hence $\lambda=0$ and $\beta$ is uniformly continuous, or $\bar{\delta}$ is bounded.

Theorem 2.1 can be reformulated in various ways. The assumption of the existence of a faithful family of $\alpha$-covariant irreducible representations is likely to follow from the approximate innerness of $\alpha$ alone. For example, if each non-zero ideal of $A$ has a non-zero projection, this follows because there exist ground states for a perturbed $\alpha$ restricted to an invariant unital hereditary $C^{*}$ subalgebra. With different arguments we can show this is also a consequence of a property of the ideal structure of the $C^{*}$-algebra.

Proposition 2.6. Let $A$ be a separable $C^{*}$-algebra and $\alpha$ an approximately inner flow. Suppose that A has at most countably many ideals. Then there exists a faithful family of $\alpha$-covariant irreducible representations of $A$.

Proof. Let $\left(h_{n}\right)$ be a sequence in $A_{s a}$ such that

$$
\alpha_{t}(x)=\lim _{n \rightarrow \infty} \operatorname{Ad} e^{i t h_{n}}(x)
$$

uniformly in $t$ on every bounded interval of $\mathrm{R}$ for all $x \in A$.

Let $\gamma$ denote the flow on $C_{0}(\mathrm{R}, A)$, the $C^{*}$-algebra of continuous functions into $A$ vanishing at infinity, induced by translation; $\gamma_{t}(x)(s)=x(s+t), x \in$ $C_{0}(\mathrm{R}, A)$ and let $\hat{\alpha}$ denote the dual action of $\mathrm{R}$ on the crossed product $A \times{ }_{\alpha} \mathrm{R}$.

We denote by $\mathrm{N}^{+}$the one-point compactification of $\mathrm{N} ; \infty$ is the newly added point. We assign the dynamical system $\left(C_{0}(\mathrm{R}, A), \gamma\right)$ to each point $n \in \mathrm{N}$ and $\left(A \times{ }_{\alpha} \mathbf{R}, \hat{\alpha}\right)$ to $\infty \in \mathrm{N}^{+}$. We assert that they form a continuous field of dynamical systems over $\mathrm{N}^{+}$.

We define a map $\phi_{n}$ of $C_{c}(\mathrm{R}, A)$, the space of continuous functions on $\mathrm{R}$ into $A$ with compact support, into $C_{0}(\mathrm{R}, A)$ by

$$
\phi_{n}(f)(p)=\int_{\mathrm{R}} d t f(t) e^{i t\left(h_{n}+p\right)}=\hat{f}\left(p+h_{n}\right) .
$$

We note that $\phi_{n}(f) \phi_{n}(g)=\phi_{n}\left(f *_{n} g\right)$ and $\phi_{n}(f)^{*}=\phi_{n}\left(f^{*_{n}}\right)$, where

$$
f *_{n} g(t)=\int_{\mathrm{R}} d s f(s) \operatorname{Ad} e^{i s h_{n}}(g(t-s))
$$

and $f^{*_{n}}(t)=\operatorname{Ad} e^{i t h_{n}}(f(-t))$. We denote by $\phi_{\infty}$ the natural embedding of $C_{c}(\mathrm{R}, A)$ into $A \times{ }_{\alpha} \mathrm{R}$, which is given by

$$
\phi_{\infty}(f)=\int_{\mathbf{R}} d t f(t) \lambda_{t},
$$


where $t \mapsto \lambda_{t}$ is the canonical unitary flow implementing $\alpha$ in the multiplier algebra of $A \times_{\alpha}$ R. We note that $\phi_{\infty}(f) \phi_{\infty}(g)=\phi_{\infty}(f * g)$ and $\phi_{\infty}(f)^{*}=$ $\phi_{\infty}\left(f^{*}\right)$, where

$$
f * g(t)=\int_{\mathrm{R}} d t f(s) \alpha_{s}(g(t-s))
$$

and $f^{*}(t)=\alpha_{t}(f(-t))$. Note that $f *_{n} g$ converges to $f * g\left(\right.$ resp. $f^{*_{n}}$ to $\left.f^{*}\right)$ uniformly as continuous functions of support contained in $\operatorname{supp}(f)+\operatorname{supp}(g)$ (resp. $-\operatorname{supp}(f))$. In particular $\left\|\phi_{n}\left(f *_{n} g\right)-\phi_{n}(f * g)\right\| \rightarrow 0$ and $\| \phi_{n}\left(f^{*_{n}}\right)-$ $\phi_{n}\left(f^{*}\right) \| \rightarrow 0$.

For $f \in C_{c}(\mathrm{R}, A)$ and $q \in \mathrm{R}$ we define $f_{q} \in C_{c}(\mathrm{R}, A)$ by $f_{q}(t)=f(t) e^{i q t}$. Then $\gamma_{q}\left(\phi_{n}(f)\right)=\phi_{n}\left(f_{q}\right)$ for $n \in \mathrm{N}$ and $\hat{\alpha}_{q}\left(\phi_{\infty}(f)\right)=\phi_{\infty}\left(f_{q}\right)$. The assertion made above comprises this fact and the following:

Lemma 2.7. If $f \in C_{c}(\mathbf{R}, A)$ then $n \in \mathbf{N}^{+} \mapsto\left\|\phi_{n}(f)\right\|$ is continuous. The range of $\phi_{n}$ is dense in $C_{0}(\mathrm{R}, A)$ if $n<\infty$ or in $A \times{ }_{\alpha} \mathrm{R}$ if $n=\infty$.

The only non-trivial claim is that $\lim _{n \rightarrow \infty}\left\|\phi_{n}(f)\right\|=\left\|\phi_{\infty}(f)\right\|$ for $f \in$ $C_{c}(\mathrm{R}, A)$. Let $\rho(f)=\lim \sup \left\|\phi_{n}(f)\right\|$. Then it follows that $\rho$ defines a $C^{*}$ seminorm on $C_{c}(\mathrm{R}, A)$ as a $*$-subalgebra of $A \times{ }_{\alpha} \mathrm{R}$. This fact follows from

$$
\begin{aligned}
\rho(f * g) & =\lim \sup \left\|\phi_{n}(f * g)\right\|=\lim \sup \left\|\phi_{n}\left(f *_{n} g\right)\right\| \\
& \leq \limsup \left\|\phi_{n}(f)\right\|\left\|\phi_{n}(g)\right\| \leq \rho(f) \rho(g), \\
\rho\left(f^{*} * f\right) & =\lim \sup \left\|\phi_{n}\left(f^{*_{n}} *_{n} f\right)\right\|=\lim \sup \left\|\phi_{n}(f)\right\|^{2}=\rho(f)^{2},
\end{aligned}
$$

etc.

Since $\rho \hat{\alpha}_{p}=\rho$ and $\rho$ is non-zero on a non-zero element $a g$ with $a \in A$ and $g \in C_{c}(\mathbf{R})$, one concludes that $\rho$ is a norm, i.e. $\rho(f)=\left\|\phi_{\infty}(f)\right\|$. Since the same statement holds for any subsequence of $\left(\phi_{n}\right)$, the claim follows.

Let $a \in A$ and $g \in C_{c}(\mathrm{R})$ and define $x \in C_{c}(\mathrm{R}, A)$ by $x(t)=a g(t)$. Then $\phi_{n}(x)(p)=a \int_{\mathrm{R}} d t g(t) e^{i t\left(h_{n}+p\right)}=a \hat{g}\left(h_{n}+p\right)$. Note that $p \mapsto\left\|\phi_{n}(x)(p)\right\|$ is a continuous function on $\mathrm{R}$ vanishing at $\infty$.

If $a \neq 0$ and $g \neq 0$, then $\phi_{\infty}(x) \neq 0$. For any $\theta \in\left\langle 0,\left\|\phi_{\infty}(x)\right\|\right\rangle$, and for all large $n$, we find the smallest $p_{n} \in \mathrm{R}$ such that $\left\|\phi_{n}(x)\left(p_{n}\right)\right\|=\theta$. Define a seminorm $\rho$ on $C_{c}(\mathrm{R}, A)$ by

$$
\rho(f)=\limsup _{n \rightarrow \infty} \max _{p \leq p_{n}}\left\|\phi_{n}(f)(p)\right\|,
$$

which extends to a $C^{*}$-seminorm on $A \times_{\alpha}$ R. Note that $\rho(x)=\theta<\left\|\phi_{\infty}(x)\right\|$ and $q \mapsto \rho \hat{\alpha}_{q}(f)$ is increasing (because $\gamma_{q} \phi_{n}(f)(p)=\phi_{n}\left(f_{q}\right)=\phi_{n}(f)(p+$ $q)$ ). 
Let

$$
\rho_{-\infty}(f)=\lim _{q \rightarrow-\infty} \rho \hat{\alpha}_{q}(f)
$$

which defines a $C^{*}$-seminorm on $A \times{ }_{\alpha}$ R.

Suppose that $\rho_{-\infty} \neq \rho$. Since $\operatorname{Ker} \rho \varsubsetneqq \operatorname{Ker} \rho_{-\infty}$, we take an irreducible representation $\pi$ of the quotient $\operatorname{Ker} \rho_{-\infty} / \operatorname{Ker} \rho$ and regard it as an irreducible representation of $A \times_{\alpha} \operatorname{R}$. Then $\operatorname{Ker} \pi \hat{\alpha}_{p} \neq \operatorname{Ker} \pi$ for $p \neq 0$; if $\operatorname{Ker} \pi \hat{\alpha}_{p}=$ $\operatorname{Ker} \pi$ for some $p \neq 0$, then $\operatorname{Ker} \pi$ contains $\operatorname{Ker} \rho \hat{\alpha}_{q}$ for any $q$ (as $\operatorname{Ker} \pi \supset$ $\operatorname{Ker} \rho$ ), from which follows that $\left.\pi\right|_{\operatorname{Ker} \rho_{-\infty}}=0$, a contradiction. This implies that the center of $\bar{\pi}$ (as defined as the direct integral of $\pi \hat{\alpha}_{p}$ over $p \in \mathbf{R}$ as before) is $L_{\infty}(\mathrm{R})$, which in turn implies that the representation $\pi$ of $A \times_{\alpha} \mathrm{R}$ is induced from an $\alpha$-covariant irreducible representation of $A$.

Suppose that $\rho_{-\infty}=\rho$, which implies that $\rho \hat{\alpha}_{q}=\rho$ for all $q$. Then there is an $\alpha$-invariant ideal $I$ of $A$ such that $\operatorname{Ker} \rho$ is described as $I \times{ }_{\alpha}$ R. For each $\theta \in\left\langle 0,\left\|\phi_{\infty}(x)\right\|\right\rangle$ we have defined a seminorm $\rho=\rho_{\theta}$ and an ideal $I=I_{\theta}$ of $A$ if $\rho_{\theta}$ is $\hat{\alpha}$-invariant. Note that if $\rho_{\theta}$ and $\rho_{\mu}$ are $\hat{\alpha}$-invariant for $\theta \neq \mu$, then $I_{\theta} \neq I_{\mu}$. If all $\rho_{\theta}$ are $\hat{\alpha}$-invariant, we thus obtain a continuous family of ideals of $A$, which contradicts the assumption. Thus there is a $\theta$ such that $\rho_{\theta}$ is not $\hat{\alpha}$-invariant.

Thus we obtain an $\alpha$-covariant irreducible representation $\pi$ of $A$. If $\operatorname{Ker} \pi$ is non-zero, we apply this argument to $\operatorname{Ker} \pi$ and $\left.\alpha\right|_{\operatorname{Ker} \pi}$, which is an approximately inner flow on a $C^{*}$-algebra with at most countably many ideals. By induction one concludes that there is a faithful family of $\alpha$-covariant irreducible representations.

\section{AF and UHF algebras}

In this section we examine some properties of cores of generators for AF and UHF algebras. First we note that if $\alpha$ is an approximately inner flow on an AFalgebra then one may choose the sequence $\left(h_{n}\right)$ which defines the generators of the approximating flows in such a way that the subspace $\mathscr{D}$ defined by (3) is dense in $A$.

Proposition 3.1. Let $A$ be an AF algebra and $\alpha$ an approximately inner flow on $A$ with generator $\delta_{\alpha}$. Then there exists a sequence $\left(h_{n}\right)$ in $A_{\text {sa }}$ such that

$$
\lim _{n \rightarrow \infty} \max _{|t| \leq 1}\left\|\alpha_{t}(x)-\operatorname{Ad} e^{i t h_{n}}(x)\right\|=0
$$

for all $x \in A$ and

$$
\mathscr{D}=\left\{x \in D\left(\delta_{\alpha}\right): \lim _{n \rightarrow \infty} \operatorname{ad} i h_{n}(x)=\delta_{\alpha}(x)\right\}
$$

is dense in A. 
REMARK 3.2. Even without assuming the existence of $\left(h_{n}\right)$ it follows from the result of Sakai mentioned in the introduction [25] that there exists an increasing sequence $A_{n}$ of finite dimensional $C^{*}$-subalgebras of $A$ such that $A_{n} \subset D\left(\delta_{\alpha}\right)$ and $\bigcup_{n} A_{n}$ is dense in $A$. This is true even if $\delta$ is not assumed to be a generator, but only a closed derivation ([7], Theorem 11). Furthermore, there exists a sequence $h_{n}$ in $A_{s a}$ such that $\left.\delta\right|_{A_{n}}=\left.\operatorname{ad}\left(i h_{n}\right)\right|_{A_{n}}$ (see [8], Example 3.2.25). If $\delta=\delta_{\alpha}$ is a generator and $\bigcup_{n} A_{n}$ is a core for $\delta_{\alpha}$ then the assertion in Proposition 3.1 follows with $\left(h_{n}\right)$ equal to this sequence since in this case $\bigcup_{n} A_{n} \subset \mathscr{D}$. In the absence of the assumption on the existence of $h_{n}$, however, it does not in general follow from the generator property of $\delta$ that $\bigcup_{n} A_{n}$ can be taken to be a core for $\delta$ or that $h_{n}$ exists (see again [20], Theorem 1.1).

Proof of Proposition 3.1. Since $\alpha$ is approximately inner there exists a sequence $d_{n}=d_{n}^{*}$ in $A_{s a}$ such that $\delta_{\alpha}$ is the graph limit of $\operatorname{ad}\left(i d_{n}\right)$. This means that for each $x$ in $D\left(\delta_{\alpha}\right)$ there exists a sequence $\left(x_{n}\right)$ of $x_{n} \in A$ such that

$$
\lim _{n \rightarrow \infty}\left\|x_{n}-x\right\|+\lim _{n \rightarrow \infty}\left\|\operatorname{ad}\left(i d_{n}\right)\left(x_{n}\right)-\delta_{\alpha}(x)\right\|=0
$$

This condition is actually equivalent to each of the equivalent conditions (1) and (2) in the introduction (see [8], Theorem 3.1.28).

To prove the proposition we next choose an increasing sequence $B_{n}$ of finite dimensional sub-algebras by Sakai's result as in the preceding remark such that $\bigcup_{n} B_{n} \subset D\left(\delta_{\alpha}\right)$. Then we are going to modify $d_{n}$ to $h_{n}$ by passing to a subsequence of $d_{n}$ such that the conclusion of Proposition 3.1 is valid.

First, fix an $n$ and let $\mathbf{X}$ be a set of matrix units for $B_{n}$ (see e.g. [12], Chapter III). Now for each matrix unit $x$ in $B_{n}$ there exists, as remarked above, a sequence $x_{m}$ in $A$ such that

$$
\lim _{m \rightarrow \infty}\left\|x_{m}-x\right\|+\lim _{m \rightarrow \infty}\left\|\operatorname{ad}\left(i d_{m}\right)\left(x_{m}\right)-\delta_{\alpha}(x)\right\|=0
$$

Using Glimm's technique we may furthermore use spectral theory to modify the approximants $x_{m}$ for each fixed $m$ such that the set of these approximants form a set of matrix units isomorphic to $\mathbf{X}$ for each $m$. This is possible if we go so far out in the sequences indexed by $m$ that the approximation to $x$ is good enough (see, for example, [12], Section III.3, or [10], Section 2). If $\mathbf{X}_{m}$ denotes the corresponding sequences of matrix units, we then have

$$
\lim _{m \rightarrow \infty}\left\|\mathbf{X}_{m}-\mathbf{X}\right\|=0
$$

However by careful scrutiny of the proof in [12] or [10] one may choose the 
approximants to $\mathbf{X}$ such that also

$$
\lim _{m \rightarrow \infty}\left\|\operatorname{ad}\left(i d_{m}\right)\left(\mathbf{X}_{m}\right)-\delta_{\alpha}(\mathbf{X})\right\|=0 .
$$

Hence

$$
\lim _{m \rightarrow \infty}\left\|\mathbf{X}_{m}-\mathbf{X}\right\|+\lim _{m \rightarrow \infty}\left\|\operatorname{ad}\left(i d_{m}\right)\left(\mathbf{X}_{m}\right)-\delta_{\alpha}(\mathbf{X})\right\|=0
$$

(The norms in these relations are defined by taking the maximum of the finite number of norms obtained by replacing the sets $\mathbf{X}$ by the individual matrix elements $x$ in $\mathbf{X}$.)

The reason for the convergence of the derivatives is that the matrix elements are obtained by applying functional calculus by smooth functions in $D(\delta)$. We use [8], Theorem 3.2.32, (or alternatively [23], or [2] and [3]) to deduce that if $\delta$ is any closed derivation and $x=x^{*}$ is in $D(\delta)$ then $f(x)$ is in $D(\delta)$. Since $f(x)$ only depends on the definition of $f$ on $\operatorname{Spec}(x)$ we may assume

$$
\left\|f\left|\|=(2 \pi)^{-1 / 2} \int_{\mathrm{R}} d p\right| \hat{f}(p)|| p \mid<\infty\right.
$$

where $\hat{f}$ is the Fourier transform of $f$. Then

$$
f(x)=(2 \pi)^{-1 / 2} \int_{\mathrm{R}} d p \hat{f}(p) e^{i p x}
$$

and

$$
\delta(f(x))=i(2 \pi)^{-1 / 2} \int_{\mathrm{R}} d p \hat{f}(p) p \int_{0}^{1} d t e^{i t p x} \delta(x) e^{i(1-t) p x} .
$$

Hence

$$
\|\delta(f(x))\| \leq\|f \mid\|\|\delta(x)\| .
$$

We need the following lemma:

Lemma 3.3. Let $\delta_{n}$ be a sequence of closed derivations on a $C^{*}$-algebra $A$ with graph limit $\delta$ and $\left(x_{n}\right)$ a sequence of elements of $A$ with $\lim _{n \rightarrow \infty} \| x_{n}-$ $x\|+\| \delta_{n}\left(x_{n}\right)-\delta(x) \|=0$. Further let $f_{n}$ be a sequence of functions converging to $f$ with respect to the semi-norm $\||\cdot|\|$.

It follows that

$$
\lim _{n \rightarrow \infty}\left\|\delta_{n}\left(f_{n}\left(x_{n}\right)\right)-\delta(f(x))\right\|=0
$$


Proof. We have

$$
\begin{aligned}
& \delta_{n}\left(f_{n}\left(x_{n}\right)\right)-\delta(f(x)) \\
& =i(2 \pi)^{-1 / 2} \int_{\mathrm{R}} d p \hat{f}_{n}(p) p \int_{0}^{1} d t e^{i t p x} \delta_{n}\left(x_{n}\right) e^{i(1-t) p x} \\
& \quad-i(2 \pi)^{-1 / 2} \int_{\mathrm{R}} d p \hat{f}(p) p \int_{0}^{1} d t e^{i t p x} \delta(x) e^{i(1-t) p x} \\
& =i(2 \pi)^{-1 / 2} \int_{\mathrm{R}} d p\left(\hat{f}_{n}(p)-\hat{f}(p)\right) p \int_{0}^{1} d t e^{i t p x} \delta_{n}\left(x_{n}\right) e^{i(1-t) p x} \\
& \quad+i(2 \pi)^{-1 / 2} \int_{\mathrm{R}} d p \hat{f}(p) p \int_{0}^{1} d t e^{i t p x}\left(\delta_{n}\left(x_{n}\right)-\delta(x)\right) e^{i(1-t) p x}
\end{aligned}
$$

from which one deduces that

(12) $\left\|\delta_{n}\left(f_{n}\left(x_{n}\right)\right)-\delta(f(x))\right\| \leq\|\| f_{n}-f\|\|\left\|\delta_{n}\left(x_{n}\right)\right\|+\|\| f\|\| \delta_{n}\left(x_{n}\right)-\delta(x) \|$.

The conclusion of the lemma follows immediately.

REMARK 3.4. The semi-norm $\|f f\|$ occurring in (10) and (12) can be estimated by noting that $|p|\left(1+p^{2}\right)^{1 / 2} \leq 2\left(1+p^{4}\right)^{1 / 2}$ and using the CauchySchwarz inequality. Hence

$$
\begin{aligned}
\|\| f \|^{2} & \leq 4\left(\int_{\mathrm{R}} d p\left(1+p^{2}\right)^{-1}\right)\left(\int_{\mathrm{R}} d p|\hat{f}(p)|^{2}\left(1+p^{4}\right)\right) \\
& =2 \pi\left(\left\|f^{\prime \prime}\right\|_{2}^{2}+\|f\|_{2}^{2}\right) .
\end{aligned}
$$

It then follows that the space of functions with $\|f\| \mid<\infty$ contains the Sobolev space $W^{2,2}(\mathrm{R})$. But these estimates are not optimal (see [2], [3], [1], [4]).

Proof of Proposition 3.1 continued. We fix a set $\mathbf{X}^{n}$ of matrix units for $B_{n}$ and suppose that we have obtained a sequence $\left(\mathbf{X}_{m}^{n}\right)$ of matrix units for each $\mathbf{X}^{n}$ by the arguments illustrated above. We then find a sequence $\left(u_{n, m}\right)$ of unitaries in $A$ such that

$$
\lim _{m \rightarrow \infty}\left\|u_{n, m}-\mathbb{1}\right\|=0
$$

and

$$
u_{n, m} \mathbf{X}^{n} u_{n, m}^{*}=\mathbf{X}_{m}^{n}
$$

where we again interpret (15) as the set of relations obtained by replacing $\mathbf{X}=\mathbf{X}_{m}^{n}, \mathbf{X}^{n}$ by each the matrix elements $x$ in $\mathbf{X}$. We can choose a subsequence 
$(m(n))$ such that $\left\|u_{n, m(n)}-\mathbb{1}\right\|<1 / n$ and $\left\|\left(\operatorname{ad}\left(i d_{m(n)}\right) \operatorname{Ad} u_{n, m(n)}-\delta\right) \mid B_{n}\right\|<$ $1 / n$. Then it follows that $\left\|\hat{\delta}_{n}(x)-\delta(x)\right\| \rightarrow 0$ for all $x \in \bigcup_{k} B_{k}$ where

$$
\hat{\delta}_{n}(x)=u_{n, m(n)}^{*} \operatorname{ad}\left(i d_{m(n)}\right)\left(u_{n, m(n)} x u_{n, m(n)}^{*}\right) u_{n, m(n)},
$$

and hence

$$
\hat{\delta}_{n}=\operatorname{ad}\left(i u_{n, m(n)}^{*} d_{m(n)} u_{n, m(n)}\right) .
$$

Since $\left\|u_{n, m(n)}-1\right\| \rightarrow 1$, one concludes that $\hat{\delta}_{n}$ converges to $\delta$ in the graph norm. This concludes the proof.

Remark 3.5. As we have already said after Proposition 3.1, if the increasing family of finite dimensional sub-algebras constitute a core for $\delta$, there is nothing more to prove. So Proposition 3.1 only tells something new when the increasing family is not a core, or $D(\delta)$ is not an AF Banach algebra in the graph norm. We do not know in general whether we can choose approximating inner derivations converging pointwise on a core for $\delta$.

The method of constructing the modified sequence again goes back to Glimm, and is expanded in Section II.3 in [12] and in Section 2 in [10].

Although Theorem 2.1 established under quite general conditions that the convergence subspace $\mathscr{D}$ cannot contain the analytic elements of the flow $\alpha$ the next example shows that there are many examples in which $\mathscr{D}$ contains an $\alpha$-invariant dense subspace of analytic elements. The following example is a flow constructed on a one-dimensional lattice. In mathematical physics terms $A$ is the algebra of observables of a one-dimensional spin- $1 / 2$ system. Note that we consider the one-dimensional case for simplicity. One can construct similar examples on higher dimensional lattices by analogous arguments.

Example 3.6. Let $A$ denote the UHF-algebra given by the $C^{*}$-closure of the infinite tensor product $\bigotimes_{n \in Z} M_{2}$ of copies of the $2 \times 2$-matrices $M_{2}$.

The algebra $A$ has a natural quasi-local structure. Let $A_{I}=\bigotimes_{i \in I} M_{2}$ denote the family of local matrix algebras indexed by finite subsets $I=\left\{i_{1}, \ldots, i_{n}\right\}$ with $i_{m} \in Z$ Z. Further let $A_{\text {loc }}=\bigcup_{I} A_{I}$. If $\sigma$ denotes the shift automorphism on $A$ then $\mathbf{Z}$ acts on $A$ as a group of shifts (space translations) $n \in \mathbf{Z} \mapsto \sigma_{n}=\sigma^{n}$ which leaves $A_{\text {loc }}$ invariant. In particular $\sigma_{n}\left(A_{I}\right)=A_{I+n}$.

Next we construct a flow corresponding to a finite-range interaction between the spins, i.e. an interaction which links close by points of Z. Fix $\Phi=\Phi^{*} \in A_{J}$ for some finite subset $J$. Define $H_{I}=H_{I}^{*}$ by

$$
H_{I}=\sum_{m \in I} \sigma_{m}(\Phi)
$$


Then introduce the corresponding inner *-derivations $\delta_{I}$ by

$$
\delta_{I}(x)=\operatorname{ad} i H_{I}(x)
$$

for all $x \in A$. Further define the *-derivation $\delta$ by $D(\delta)=A_{\text {loc }}$ and

$$
\delta(x)=\lim _{I} \operatorname{ad} i H_{I}(x)
$$

for $x \in A_{\text {loc }}$ where the limit is over an increasing family of $I$ whose union is $Z$. (For each $x \in A_{\text {loc }}$ there is an $I \in Z$ such that $\delta(x)=\operatorname{ad} i H_{I}(x)$ by locality.)

There is a flow $\alpha$ on $A$ given by

$$
\alpha_{t}(x)=\lim _{I} \operatorname{Ad} e^{i t H_{I}}(x) .
$$

The norm limit exists by the estimates of [9], Theorem 6.2.4. Moreover the generator $\delta_{\alpha}$ of the flow is the norm closure $\bar{\delta}$ of the derivation $\delta$. Then $\delta_{\alpha}$ is the graph limit of the derivations $\delta_{I}$ (see [8], Theorem 3.1.28). Therefore if we define

$$
\mathscr{D}=\left\{x \in D\left(\delta_{\alpha}\right): \lim _{I} \delta_{I}(x)=\delta_{\alpha}(x)\right\}
$$

one has

$$
A_{\mathrm{loc}}=D(\delta) \subseteq \mathscr{D} \subseteq D\left(\delta_{\alpha}\right) .
$$

Finally define $\mathscr{A}$ as the ${ }^{*}$-algebra generated by $\left\{\alpha_{t}\left(A_{\text {loc }}\right): t \in \mathrm{R}\right\}$. Then we argue that $\mathscr{A} \subseteq \mathscr{D}$. To this end it suffices to show that if $x \in A_{J}$ for some $J$ and $t \in \mathrm{R}$ then $\lim _{I}$ ad $H_{I}\left(\alpha_{t}(x)\right)$ exists. But if $I_{1} \subset I_{2}$ then

$$
\text { ad } H_{I_{1}}\left(\alpha_{t}(x)\right)-\operatorname{ad} H_{I_{2}}\left(\alpha_{t}(x)\right)=\sum_{p \in I_{2} \backslash I_{1}}\left[\sigma_{p}(\Phi), \alpha_{t}(x)\right] .
$$

Then, as a consequence of [9], Proposition 6.2.9, there are $a, b, c>0$ such that

$$
\left\|\left[\sigma_{p}(\Phi), \alpha_{t}(x)\right]\right\| \leq a\|x\| e^{-b p+c t}
$$

uniformly for $p \in \mathbf{Z}$ and $t \in \mathbf{R}$. Therefore

$$
\left\|\operatorname{ad} H_{I_{1}}\left(\alpha_{t}(x)\right)-\operatorname{ad} H_{I_{2}}\left(\alpha_{t}(x)\right)\right\| \leq a\|x\| e^{c t} \sum_{p \in I_{1}^{\mathrm{c}}} e^{-b p}
$$

for all $t \in \mathrm{R}$. It follows immediately that the limit exists and this suffices to establish the inclusion $\mathscr{A} \subseteq \mathscr{D}$.

One concludes that $\mathscr{A}$ is an $\alpha$-invariant subspace of $D\left(\delta_{\alpha}\right)$. Therefore it is a core of $\delta_{\alpha}$. But it also follows from [9], Theorem 6.2.4, that each $x \in \mathscr{A}$ is 
an analytic element of $\delta_{\alpha}$. Thus $\mathscr{D}$ contains an $\alpha$-invariant dense subalgebra of analytic elements.

Since the flow $\alpha$ commutes with the group of translations by $\mathrm{Z}$ it follows that the Connes' spectrum $\mathbf{R}(\alpha)$, which is a subgroup of $\mathbf{R}$, must be $\operatorname{Sp}(\alpha)$. Therefore $\mathbf{R}(\alpha) \cong \mathbf{Z}$ or $\mathbf{R}$ if $\alpha$ is non-trivial. Both cases can occur with a suitable choice of $\Phi$.

Although the latter conclusions rely on translation invariance one can construct similar examples on the half line and the same conclusions are valid. In particular one can add a bounded ${ }^{*}$-derivation $\delta_{P}$ to $\delta_{\alpha}$ in such a way that $\alpha$ factors into a product of flows $\alpha^{( \pm)}$on the left and right half lattice $\mathbf{Z}_{ \pm}$, respectively. Then the algebra $\mathscr{A}^{+}$generated by $\left\{\alpha_{t}^{(+)}\left(A_{I}\right): t \in \mathrm{R}, I \subset \mathrm{Z}_{+}\right\}$ is contained in the set $\mathscr{D}\left(\alpha^{(+)}\right)$corresponding to $\alpha^{(+)}$and consists of analytic elements for the latter flow. Finally $\mathrm{R}\left(\alpha^{(+)}\right)=\mathrm{R}(\alpha)$ because $\alpha^{(-)} \otimes \alpha^{(+)}$arises by a bounded perturbation of the generator of $\alpha$.

The next example shows that $\mathscr{D}$ can be much larger but then the Connes' spectrum is equal to $\{0\}$.

Example 3.7. Let $A$ denote the UHF-algebra given by the $C^{*}$-closure of the infinite tensor product $\bigotimes_{n \geq 1} M_{2}$ of copies of the $2 \times 2$-matrices $M_{2}$, $A_{I}=\bigotimes_{i \in I} M_{2}$ the local matrix algebras and $A_{\mathrm{loc}}=\bigcup_{I} A_{I}$.

Now let $\left(\lambda_{i}\right)_{i \geq 1}$ be a sequence of positive numbers and define $h_{i} \in A_{\{i\}}$ by

$$
h_{i}=\left(\begin{array}{cc}
\lambda_{i} & 0 \\
0 & 0
\end{array}\right) .
$$

Set $H_{n}=\sum_{i=1}^{n} h_{i}$ and $\delta_{n}(x)=\operatorname{ad} i H_{n}(x)$ for $x \in A$. Then define $\alpha$ on $A$ by

$$
\alpha_{t}(x)=\lim _{I} \operatorname{Ad} e^{i t H_{I}}(x) .
$$

Set

$$
\mathscr{D}=\left\{x \in D\left(\delta_{\alpha}\right): \lim _{I} \delta_{I}(x)=\delta_{\alpha}(x)\right\} .
$$

We next argue that if the $\lambda_{i}$ are chosen to increase sufficiently fast as $i \rightarrow \infty$ then $D\left(\delta_{\alpha}^{2}\right) \subset \mathscr{D}$ but in this case $\mathrm{R}(\alpha)=\{0\}$.

Let $a_{i}, a_{i}^{*} \in A_{\{i\}}$ be given by

$$
a_{i}=\left(\begin{array}{cc}
0 & 1 \\
0 & 0
\end{array}\right) \quad \text { and } \quad a_{i}^{*}=\left(\begin{array}{cc}
0 & 0 \\
1 & 0
\end{array}\right)
$$

and note that $H_{n}=\sum_{i=1}^{n} \lambda_{i} a_{i}^{*} a_{i}$. Set $a(I)=\bigotimes_{i \in I} a_{i}$ and $a^{*}(J)=\bigotimes_{j \in J} a_{j}^{*}$.

Let $C_{2}$ be the diagonal matrices of $M_{2}$ and $C$ the $C^{*}$-subalgebra generated by $C_{2}$ at every point of $\mathrm{N} ; C=\bigotimes_{i \in \mathrm{N}} C_{2} \subset A=\bigotimes_{i \in \mathrm{N}} M_{2}$. For a subset $K$ of $\mathrm{N}$ let $C_{K}=\bigotimes_{i \in K} C_{2}$. 
We define an action $\gamma$ of $G=\prod_{n=1}^{\infty} \mathrm{T}$ on $A$ by

$$
\gamma_{z}=\bigotimes_{n=1}^{\infty} \operatorname{Ad}\left(\begin{array}{cc}
z_{n} & 0 \\
0 & 1
\end{array}\right)
$$

Then the fixed point algebra of $\gamma$ is $C$ while $a(I)$ and $a^{*}(J)$ are eigenoperators for finite subsets $I, J: \gamma_{z}(a(I))=\prod_{n \in I} z_{n} a(I)$. The spectrum of $\gamma$ is $\coprod\{-1,0,1\} \subset \hat{G}=\coprod_{n \in \mathrm{N}} \mathrm{Z}$, which we identify with $\mathscr{S}=\{(I, J) \in$ $\left.P_{f}(\mathrm{~N}) \times P_{f}(\mathrm{~N}) \mid I \cap J=\emptyset\right\}$, where $P_{f}(\mathrm{~N})$ is the set of finite subsets of $\mathrm{N}$ and $p \in \coprod\{-1,0,1\}$ maps to $(I, J)$ with $I=\left\{n \mid p_{n}=-1\right\}$ and $J=\left\{n \mid p_{n}=1\right\}$. Note that for each $(I, J) \in \mathscr{S}$ the eigen-space is given by $C a(I) a^{*}(J)=C_{I^{c} \cap J^{c}} a(I) a^{*}(J)$.

Then each $x \in A_{\text {loc }}$ has a unique representation

$$
x=\sum_{(I, J) \in \mathscr{S}} x(I ; J) a^{*}(I) a(J)
$$

with $x(I ; J) \in C_{I^{c} \cap J^{c}}$, where the sum is finite. Let $z^{I}=\prod_{n \in I} z_{n}$ and $\bar{z}^{J}=$ $\prod_{n \in J} \bar{z}_{n}$. Since

$$
\int_{G} z^{I} \bar{z}^{J} \gamma_{z}(x) d z=x(I, J) a^{*}(I) a(J)
$$

with $d z$ is normalized Haar measure on $G$, one deduces that $\|x(I ; J)\| \leq\|x\|$.

Now $x \in D\left(\delta_{\alpha}^{2}\right)$ by locality,

$$
\delta_{\alpha}(x)=i \sum_{(I, J) \in \mathscr{S}}(\lambda(I)-\lambda(J)) x(I ; J) a^{*}(I) a(J)
$$

where $\lambda(I)=\sum_{i \in I} \lambda_{i}$ and

$$
\delta_{\alpha}^{2}(x)=-\sum_{(I, J) \in \mathscr{S}}(\lambda(I)-\lambda(J))^{2} x(I ; J) a^{*}(I) a(J) .
$$

In particular $(\lambda(I)-\lambda(J))^{2}\|x(I ; J)\| \leq\left\|\delta_{\alpha}^{2}(x)\right\|$.

Next suppose $\lambda_{n} \geq 2\left(\lambda_{1}+\cdots+\lambda_{n-1}\right)+6^{n}$ for all $n$. If $n=\max (I \cup J)$ then

$$
|\lambda(I)-\lambda(J)| \geq \lambda_{n}-\left(\lambda_{1}+\cdots+\lambda_{n-1}\right) \geq 6^{n} .
$$


Therefore $6^{n}|\lambda(I)-\lambda(J)|\|x(I ; J)\| \leq\left\|\delta_{\alpha}^{2}(x)\right\|$ and

$$
\begin{aligned}
\left\|\delta_{\alpha}(x)\right\| & \leq \sum_{(I, J) \in \mathscr{S}}|\lambda(I)-\lambda(J)|\|x(I ; J)\| \leq \sum_{n \geq 1} \sum_{\max (I \cup J)=n} 6^{-n}\left\|\delta_{\alpha}^{2}(x)\right\| \\
& \leq \sum_{n \geq 1} 2^{-n}\left\|\delta_{\alpha}^{2}(x)\right\|=\left\|\delta_{\alpha}^{2}(x)\right\|
\end{aligned}
$$

where we have used $\sum_{\max (I \cup J)=n} 1<3^{n}$. But $A_{\text {loc }}$ is a core of $\delta_{\alpha}^{2}$. The foregoing estimates then establish that the representation (18) extends to all $x \in D\left(\delta_{\alpha}^{2}\right)$; the infinite sum in (18) is absolutely convergent.

Then if $k \leq l$ one has $H_{l}-H_{k}=\sum_{i=k+1}^{l} \lambda_{i} a_{i}^{*} a_{i}$ and so

$$
\delta_{l}(x)-\delta_{k}(x)=i \sum_{I, J}\left(\lambda_{k, l}(I)-\lambda_{k, l}(J)\right) x(I ; J) a^{*}(I) a(J)
$$

for all $x \in D\left(\delta_{\alpha}^{2}\right)$ with $\lambda_{k, l}(I)=\lambda(I \cap\{k+1, \ldots, l\})$. In particular the summand is only non-zero if $\max (I \cup J)>k$. But $\lambda_{k, l}(I)=\lambda_{l}(I)-\lambda_{k}(I)$ with $\lambda_{l}(I)=\lambda(I \cap\{1, \ldots, l\})$. Now if $n+1 \in I \cup J$ then

$$
\left|\lambda_{n+1}(I)-\lambda_{n+1}(J)\right| \geq \lambda_{n+1}-\left(\lambda_{1}+\cdots+\lambda_{n}\right)
$$

and

$$
\left|\lambda_{n}(I)-\lambda_{n}(J)\right| \leq \lambda_{1}+\cdots+\lambda_{n} .
$$

Hence

$$
\left|\lambda_{n}(I)-\lambda_{n}(J)\right| \leq\left|\lambda_{n+1}(I)-\lambda_{n+1}(J)\right| .
$$

But if $n+1 \notin I \cup J$ then $\lambda_{n}(I)-\lambda_{n}(J)=\lambda_{n+1}(I)-\lambda_{n+1}(J)$ so (20) is valid for all $n$. Then by iteration

$$
\left|\lambda_{k}(I)-\lambda_{k}(J)\right| \leq\left|\lambda_{l}(I)-\lambda_{l}(J)\right| \leq|\lambda(I)-\lambda(J)| .
$$

Combining these observations one concludes from (19) that

$$
\left\|\delta_{l}(x)-\delta_{k}(x)\right\| \leq \sum_{\max (I \cup J)>k}|\lambda(I)-\lambda(J)|\|x(I ; J)\| \leq 2^{-k}\left\|\delta_{\alpha}^{2}(x)\right\|
$$

for all $x \in D\left(\delta_{\alpha}^{2}\right)$. Therefore $\delta_{l}(x) \rightarrow \delta_{\alpha}(x)$ as $l \rightarrow \infty$ and $\mathscr{D} \supseteq D\left(\delta_{\alpha}^{2}\right)$.

Note that in this example $\mathrm{R}(\alpha)=\{0\}$ but $\delta_{\alpha}$ is not bounded.

ACKNOWLEDGEMENTS. This work was carried out whilst the first and second authors were visiting the Centre for Mathematics and its Applications at the Australian National University, Canberra. 


\section{REFERENCES}

1. van Ackooij, W., de Pagter, B., and Sukochev, F. A., Domains of infinitesimal generators of automorphism flows, J. Funct. Anal. 218 (2005), 409-424.

2. Arazy, J., Barton, T. J., and Friedman, Y., Operator differentiable functions, Integral Equations Operator Theory 13 (1990), 461-487.

3. Arazy, J., Barton, T. J., and Friedman, Y., Addendum to [2], Integral Equations Operator Theory 14 (1991), 889.

4. Arazy, J., and Zelenko, L., Directional operator differentiability of non-smooth functions, J. Operator Theory 55 (2006), 49-90.

5. Batty, C. J. K., and Kishimoto, A., Derivations and one-parameter subgroups of $C^{*}$-dynamical systems, J. London Math. Soc. 31 (1985), 526-536.

6. Bratteli, O., Kishimoto, A., and Robinson, D. W., Embedding product type actions into $C^{*}$ dynamical systems, J. Funct. Anal. 75 (1987), 188-210.

7. Bratteli, O., and Robinson, D. W., Unbounded derivations of $C^{*}$-algebras, Comm. Math. Phys. 42 (1975), 253-268.

8. Bratteli, O., and Robinson, D. W., Operator Algebras and Quantum Statistical Mechanics, vol. 1. Second edition. Second printing. Springer-Verlag, New York etc., (2002).

9. Bratteli, O., and Robinson, D. W., Operator Algebras and Quantum Statistical Mechanics, vol. 2. Second edition. Second printing. Springer-Verlag, New York etc., (2002).

10. Bratteli, O., Inductive limits of finite dimensional $C^{*}$-algebras, Trans. Amer. Math. Soc. 171 (1972), 195-234.

11. Bratteli, O., Derivations, Dissipations and Group Actions on $C^{*}$-algebras, Lecture Notes in Math. 1229 (1986).

12. Davidson, K. R., $C^{*}$-algebras by Example,Fields Inst. Monogr. 6 (1996).

13. Dixmier, J., and Malliavin, P., Factorisations de fonctions et de vecteurs indéfiniment différentiables, Bull. Sci. Math. (2) 102 (1978), 305-330.

14. Kato, T., Perturbation Theory for Linear Operators. Second edition, Grundlehren Math. Wiss. 132 (1984).

15. Kishimoto, A., Derivations with a domain condition, Yokohama Math. J. 32 (1984), 215-223.

16. Kishimoto, A., Type I orbits in the pure states of a $C^{*}$-dynamical system, Publ. Res. Inst. Math. Sci. 23 (1987), 321-336.

17. Kishimoto, A., Type I orbits in the pure states of a $C^{*}$-dynamical system II, Publ. Res. Inst. Math. Sci. 23 (1987), 517-526.

18. Kishimoto, A., Automorphism groups and covariant irreducible representations, in Mappings of Operator algebras, H. Araki, R. V. Kadison eds. Birkhauser, 1990, 129-139.

19. Kishimoto, A., Quasi-product flows on a $C^{*}$-algebra, Comm. Math. Phys. 229 (2002), 397 413.

20. Kishimoto, A., Non-commutative shifts and crossed products, J. Funct. Anal. 200 (2003), 281-300.

21. Kishimoto, A., and Robinson, D. W., Derivations, dynamical systems, and spectral restrictions, Math. Scand. 56 (1985), 83-95.

22. Kissin, E., and Shulman, V., Representations on Krein Spaces and derivations on $C^{*}$-algebras, Pitman Monogr. Surveys Pure Appl. Math. 89 (1997).

23. Pedersen, G. K., Operator differentiable functions, Publ. Res. Inst. Math. Sci. 36 (2000), 139-157.

24. Rubel, L. A., Squires, W. A., and Taylor, B. A., Irreducibility of certain entire functions with applications to harmonic analysis, Ann. of Math. 108 (1978), 553-567.

25. Sakai, S., On one-parameter groups of automorphisms on operator algebras and the corresponding unbounded derivations, Amer. J. Math. 98 (1976), 427-440. 
26. Sakai, S., Operator Algebras in Dynamical Systems, Encyclopedia Math. Appl. 41, Cambridge Univ. Press, 1991.

DEPARTMENT OF MATHEMATICS

UNIVERSITY OF OSLO

BLINDERN

P.O.BOX 1053

N-0316 NORWAY

E-mail: bratteli@math.uio.no

CENTRE FOR MATHEMATICS AND ITS APPLICATIONS

AUSTRALIAN NATIONAL UNIVERSITY

CANBERRA ACT 0200

AUSTRALIA
DEPARTMENT OF MATHEMATICS HOKKAIDO UNIVERSITY

SAPPORO 060-0810

JAPAN 